2. to examine the methodological opportunities and challenges of using story completion to identify cultural narratives.

Methods Story completion is a radically different approach to collecting qualitative data to explore social constructions concerning a certain topic and the socio-cultural discourses and dominant meanings available to participants. Story completion research involves participants being provided with the opening sentences of a hypothetical scenario - or story stem - and asked to write what happens next. Four story stems were designed and refined based upon a pilot study and feedback from experts in qualitative methods and disability. Ninety UKbased undergraduate sport and exercise science students wrote a story in response to one of the stems, randomly allocated. The stories were subject to a thematic and structural narrative analysis.

Results Analysis is underway. The supercrip narrative was the most dominant narrative type drawn upon, with interwoven elements of tragedy and romance from a fairy-tale narrative. The less prescriptive story stems elicited the more diverse and creative stories in terms of length and detail.

Conclusions The need for alternative stories of physical activity and disability that challenge the underlying ableist assumptions of the dominant supercrip narrative will be discussed. Reflections of story completion will be provided with recommendations for future use.

\section{O36 USING STORY COMPLETION TASKS TO EXPLORE PERCEPTIONS ABOUT MENTAL HEALTH IN A WORK CONTEXT}

${ }^{1}$ Irmgard Tischner, ${ }^{2}$ Naomi Moller, ${ }^{2}$ Andreas Vossler. ${ }^{1} \mathrm{TH}$ Deggendorf, Deggendorf, Germany; ${ }^{2}$ The Open University, Milton Keynes, UK

\subsection{6/bmjopen-2019-QHRN.36}

Background Increasing numbers of people struggle with mental health issues in western industrial nations, including the UK and Germany, with about a quarter of the population experiencing mental health problems in the space of a year (WHO/ Europe, 2018). In neoliberal cultures, individuals are often positioned as responsible for their psychological (and physical) health. This is evidenced, in part, by the proliferation of resilience, stress management, and similar self-help courses. Such courses and resources focus on teaching individuals how to cope with mental health issues, disregarding potential social, cultural and environmental causes for psychological distress.

Aims/Objectives Considering this, we explored how this individualising of mental health plays out in the context of the work place, in particular in the discourses drawn on in narratives about colleagues who experience psychological illness or distress.

Methods We collected data using the method of story completion tasks (Clarke et al., 2017), asking participants to complete the following story stem, with half of participants receiving the stem with a female and half with a male protagonist: 'Michael/a returns to work after 3 weeks off. In the meantime word had got around that s/he hadn't been on annual leave, but signed off sick with mental health issues. Please continue Michael/a's story.' Participants were individuals working in either Germany or the UK, who completed the task via an online survey platform.

Results and conclusion The study is still in the early stages. We will report thematic/discursive analysis around constructions of mental health issues in general, the social perceptions about individuals with mental health issues, as well as their positioning in the context of the workplace reflected in the narratives told. We also reflect on the value of story completion for exploring understandings around workplace mental health.

\section{REFERENCES}

1. Clarke V, Hayfield N, Moller N, et al. (2017) Once upon a time...: Qualitative story completion methods. In: Braun, V., Clarke, V. and Gray, D., eds. (2017) Collecting Qualitative Data: A Practical Guide to Textual, Media and Virtual Techniques. Cambridge: Cambridge University Press. ISBN 9781107054974. Available from: http://eprints.uwe.ac.uk/29937.

2. WHO Regional Office for Europe (2018) http://www.euro.who.int/en/health-topics/ noncommunicable-diseases/mental-health/data-and-resources (accessed September 2018).

\section{Poster presentations}

\section{P1 NOTIONS OF SAFETY AND RISK IN PALLIATIVE CARE: THE ROLE OF QUALITATIVE HEALTH RESEARCH} ${ }^{1,2}$ Sarah Yardley, ${ }^{3}$ Huw Williams, ${ }^{4}$ Simon Noble, ${ }^{4}$ Annmarie Nelson, ${ }^{3}$ Toby Dinnen,
${ }^{5}$ Liam Donaldson, ${ }^{3}$ Andrew Carson-Stevens. ${ }^{1}$ Central and North West London NHS
Foundation Trust, London, UK; ${ }^{2}$ Marie Curie Research Department, University College
London, London, UK; ${ }^{3}$ Division of Population Medicine, Cardiff University, Cardiff, UK;
${ }^{4}$ School of Medicine, Cardiff University, Cardiff, UK; ${ }^{5}$ London School of Hygiene and Tropical
Medicine, London, UK

\subsection{6/bmjopen-2019-QHRN.37}

Background Technological advances and societal expectations are transforming professional-patient relationships, while interpersonal therapeutic interactions remain essential to palliative care. ${ }^{1,2,3}$ Harm can be experienced as an 'unfolding series of negative events...inextricably linked with feeling unsafe'. Negotiating safe care that remains sensitive to patients' priorities can create tensions with safety standards, while failure to consider these can result in avoidable harms including worsened symptoms and disrupted dying. . $^{5,6}$

Aims Demonstrate how QHR can deepen understanding of safety and risk in palliative care.

Showcase use of social research methods to identify implications for professional learning by integrating incident analysis with multi-voiced constructions of interpersonal safety work. $^{4,7}$

Methods We developed innovative mixed method combinations of systematic review techniques with cross-sectional quantitative descriptive analysis and interpretative qualitative meta-syntheses of data from UK National incident reporting systems, stakeholder perspectives and published literature. ${ }^{8,9}$ Details will be given with each case study presented.

Results QHR enables professional learning about insights into everyday safety challenges and realities for patients, informal carers and professional working practices. Patients are vulnerable not only to generic safety risks but also additional risks specific to the last phase of life. For example, many harms are mediated through medication work with problematic consequences. Advance care planning (ACP) is often postulated as a solution to care challenges. Our qualitative analysis of ACP incidents demonstrates safe ACP requires not simply theoretical knowledge but acceptance of socially constructed roles and responsibilities with applied metacognitive skills and emotional intelligence. We present a new analysis of intersectionality between enactment of patient, carer and professional 